\title{
The Role of Calcium in the Rapid Adaptation of an Insect Mechanoreceptor
}

\author{
A. S. French \\ Department of Physiology, University of Alberta, Edmonton, Alberta T6G 2H7, Canada
}

The femoral tactile spine of the cockroach is a rapidly adapting mechanoreceptor in which the response to a step displacement is a burst of action potentials lasting about 1 sec. This adaptation seems to occur during the encoding of action potentials from receptor current, since there is no evidence of adaptation in the receptor potential, and the adaptation can be demonstrated by direct electrical stimulation of the single sensory neuron in the spine.

We have recently presented evidence for a calcium-activated potassium conductance in the tactile spine receptor neuron, although it was not directly linked to adaptation. Since this conductance could be involved in the rapid adaptation of the tactile spine neuron, we sought an association between adaptation and the entry of calcium ions into the cell. Adaptation in the tactile spine neuron was examined by direct electrical stimulation using a randomly fluctuating current followed by frequency-response analysis. The dynamic behavior was studied as a function of the concentration of calcium ions in the bathing solution, the presence of calcium channel blocking agents, or the divalent cation ionophore antibiotic A23187. No evidence could be found that entry of calcium ions into the cell has a major role in the sensory adaptation.

The cockroach femoral tactile spine is a Type $I$, or cuticular, arthropod mechanoreceptor (McIver, 1975; Rice, 1975) containing a single bipolar sensory neuron, located in the base of the spine and sending its axon through the femur into the CNS (French and Sanders, 1981). Movement of the spine produces a burst of action potentials that typically adapts completely in about $1 \mathrm{sec}$. This rapid adaptation cannot be characterized by a single-exponential process (Pringle and Wilson, 1952) but can be well fitted by a power-law function (Chapman and Smith, 1963). Examination of the receptor current in the tactile spine neuron did not show any adaptation during the transduction of mechanical stimuli (French, 1984b), indicating that adaptation must, instead, occur when the receptor current is cncoded into action potentials. This suggestion was confirmed by the finding that the response of the sensory neuron to direct electrical stimulation showed a rapid adaptation with dynamic properties identical to those seen with mechanical stimulation (French, $1984 a, c)$.

Although adaptation of action potential discharge to a maintained stimulus is found in a wide variety of neurons, there is no coherent picture of the mechanisms underlying it. Some of the processes that could be responsible include changes in the activation or inactivation of sodium and potassium channels, accumulation of ions inside or outside the neuronal membrane,

Received Aug. 22, 1985; revised Jan. 20, 1986; accepted Jan. 25, 1986.

This work was supported by grants from the Medical Research Council of Canada and the Alberta Ieritage Foundation for Mcdical Research. I would like to thank Rodney Gramlich for expert technical assistance throughout.

Correspondence should be addressed to Andrew S. French at the above address. Copyright (C) 1986 Society for Neuroscience $0270-6474 / 86 / 082322-05 \$ 02.00 / 0$ activation of an electrogenic pump, and opening of potassium channels by the entry of calcium ions during depolarization (Jack et al., 1983). The last of these mechanisms suggests that depolarization, with or without action potentials, leads to calcium ion entry, and this in turn activates a potassium conductance, which tends to reduce excitability by hyperpolarizing the membrane and increasing its conductance. This process has been strongly implicated in the adaptation of several neurons, including some sensory receptors (Jansen and Nicholls, 1973; Lewis and Wilson, 1982; Ottoson and Swerup, 1982; Partridge and Stevens, 1976). A calcium-activated potassium conductance has been described in cockroach central neurons (Thomas, 1981), and we have recently presented evidence for an afterhyperpolarization following bursts of action potentials in the cockroach tactile spine neuron that can be eliminated by calcium blocking chemicals (French, 1985a). However, the power-law behavior of the tactile spine neuron, which describes its adaptation properties, can also be seen in the threshold sensitivity to sinusoidal inputs (French, 1984c). At high frequencies $(\sim 100$ $\mathrm{Hz}$ ), the threshold current is very small and may cause a depolarization of only a few millivolts (French, 1984a). This made us cautious of ascribing the adaptation solely to a calciumactivated potassium conductance, which might require significant depolarization. We therefore sought in the present experiments to determine if calcium entry is crucial to rapid adaptation. This was tested by varying the concentration of calcium ions in the external solution, by applying inorganic calcium blocking agents, and by applying a calcium ionophore. We found no evidence that calcium entry is important for adaptation.

\section{Materials and Methods}

Cockroaches, Periplaneta americana, were maintained in a laboratory colony. Femoral tactile spines on the right metathoracic legs of adult animals were used. Legs were amputated distal to the trochanter and mounted in a Plexiglas chamber. Care was taken to ensure that the main trachea in the femur was open, and the chamber design ensured that the cut end was exposed to the air. Two insect pins were passed through the femur at a distance of $5 \mathrm{~mm}$ from the spine and close to nerve $5 \mathrm{r} 8 \mathrm{e}$. The pins served as extracellular recording electrodes for afferent activity and were connected to a differential AC amplifier.

A Vaseline dam was constructed around the spine and filled with cockroach saline solution of composition (mM): $\mathrm{NaCl}, 124 ; \mathrm{KCl}, 10$; $\mathrm{CaCl}_{2}, 5 ; \mathrm{MgCl}_{2}, 1$; MOPS, 3; NaMOPS, 3.9; sucrose, $40 ; \mathrm{pH} 7.2$ (Chesler and Fourtner, 1981). The spine was cut off just distal to the sensory neuron and a glass microelectrode was lowered into the lumen of the spine and adjacent to the sensory axon (Fig. 1). Microelectrodes were filled with $0.5 \mathrm{M} \mathrm{NaCl}$ and had resistances of about $5 \mathrm{M} \Omega$. The microelectrode was connected to the stimulating circuit by a $\mathrm{Ag} / \mathrm{AgCl}$ electrode, and the cut end of the tibia was immersed in saline together with an $\mathrm{Ag} / \mathrm{AgCl}$ return electrode connected to a virtual ground circuit to measure current flow. At the start of each experiment the position of the electrode was moved to the position of minimum threshold current for a $1 \mathrm{~Hz}$ sinusoidal stimulus.

Pseudo-random noise was generated from a 33-bit shift register clocked at $1 \mathrm{kHz}$ (Marmarelis and Marmarelis, 1978) and band-limited to 0$125 \mathrm{~Hz}$ by a 9-pole active filtcr. The noise signal was used to drive a 


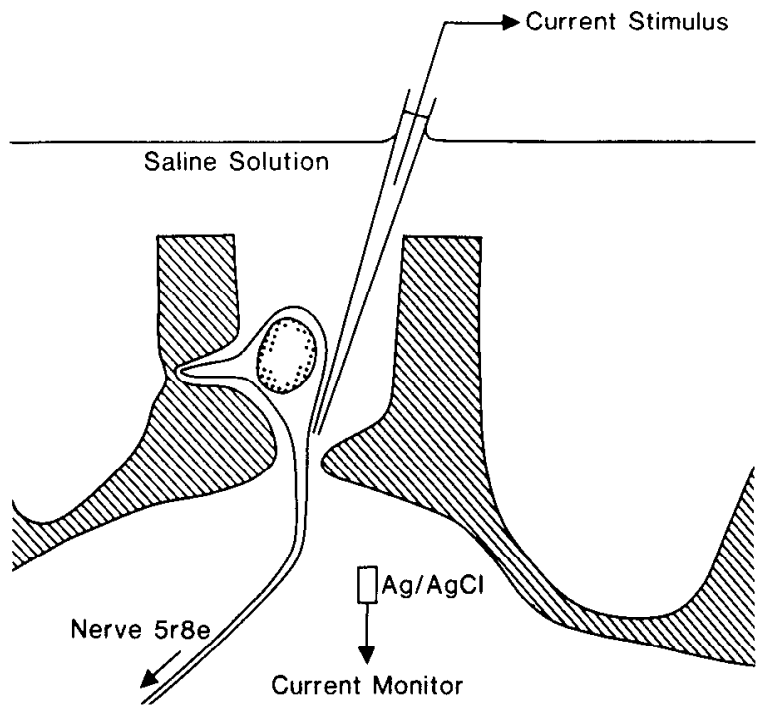

Figure 1. Experimental arrangement for electrical stimulation of the sensory neuron in the tactile spine. Microelectrodes were advanced down the lumen of the cut spine and placed in the position of maximum stimulus sensitivity, which was always close to the base of the spine. $\mathrm{The} \mathrm{Ag} / \mathrm{AgCl}$ electrode for the current monitor was actually located at the cut end of the tibia. Action potentials were observed by extracellular electrodes adjacent to the axon in the femur.

randomly varying stimulus current through the microelectrode. The current signal was sampled at $4 \mathrm{msec}$ intervals by a 12-bit analog-todigital converter connected to a PDP- 11 computer. The current stimulus was adjusted to an amplitude of approximately $23 \mathrm{nA}$ root mean square (rms) at the start of cach expcriment. The final accurate measure of stimulus amplitude was obtained from the input spectrum at the end of the experiment. The afferent action potentials were detected with a Schmitt trigger connected to the AC amplifier, and the resultant pulses were also sent to the computer. Action potentials were observed with a time resolution of $100 \mu \mathrm{sec}$ and digitally filtered to a bandwidth of 0 $125 \mathrm{~Hz}$ (French and Holden, 1971). The rate of action potential firing was calculated at $10 \mathrm{sec}$ intervals during the experiment by means of a digital counter. The frequency-response function between current stimulus and action potential output was computed by taking the fast Fourier transform of the 2 signals and then generating the input, output, and cross spectra, using software similar to that described previously (French, 1973). Frequency-response functions were displayed as Bode plots of phase and $\log$ gain versus $\log$ frequency. Measurement of each frequency-response function required approximately $3 \mathrm{~min}$ of stimulation with random noise. At other times, the stimulus was removed and the preparation was silent.

Adaptation in the tactile spine neuron can be characterized as a power law of time, following a step input, or as a power law of frequency when the frequency-response function is measured. These 2 power laws can be related to each other using linear systems theory (Chapman and Smith, 1963; French, 1984a; French et al., 1972). Experimentally, the frequency-response measurement using a band-limited white noise stimulus offers the most efficient means of examining the behavior and leads to a response of the following form:

$$
Y(j \omega)=G \cdot(j \omega)^{k}
$$

where $Y(j \omega)$ is the frequency-response function, $\omega$ is radial frequency, $j=\sqrt{-1}, G$ is the gain at $\omega=1 \mathrm{radian} / \mathrm{sec}$, and $k$ is a fractional exponent of frequency that defines the adaptation characteristics of the receptor. Note that $k=0$ for a system that does not adapt and $k=1$ for a system that behaves as a differentiator and therefore adapts rapidly. The parameter $G$ indicates the sensitivity of the preparation. The Bode plot of $\log$ gain versus $\log$ frequency is linear with slope $k$ and intercept $\log G$. In the tactile spine preparation, the exponent and gain vary with the amplitude of the noise stimulus, the mean membrane potential of the neuron (French, 1984a), and temperature (French, 1985b). In the present experiments the stimulus amplitude was held as constant as possible at about $23 \mathrm{nA}(\mathrm{rms})$, and the temperature of the preparation was held

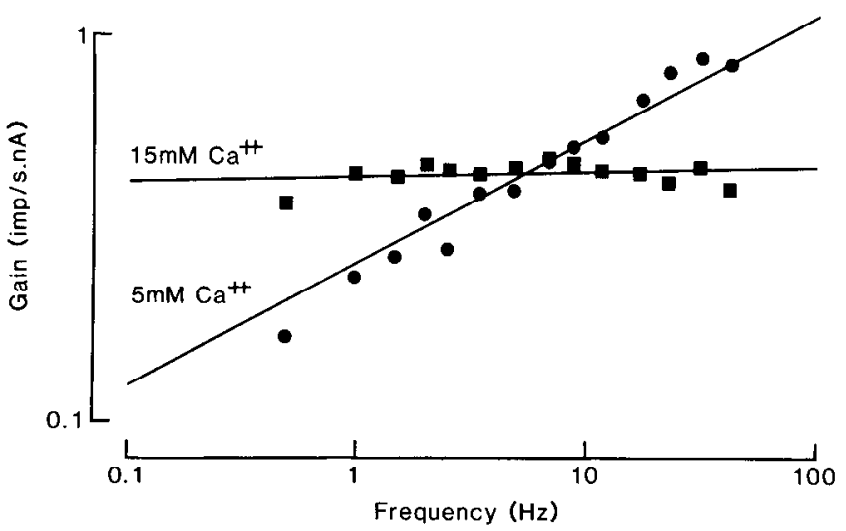

Figure 2. Gain portions of two frequency-response functions for encoding of action potentials by a tactile spine neuron, in salines containing normal and elevated calcium concentrations. The fitted lines represent the power law of equation (1) with parameters $k=0.35, G=0.15$ in normal saline, and $k=0.15, G=0.49$ in high calcium. Phase data were also plotted for all frequency-response functions and were well fitted by the power law, after accounting for the conduction delay to the recording electrodes.

constant to within $1^{\circ} \mathrm{C}$. The mean current through the electrode was held at zero to avoid changing the mean membrane potential.

Changes in the ionic composition of the bathing solution were balanced by varying the amount of sucrose to maintain the original osmolarity. Chemical agents used were cobalt chloride and cadmium chloride (Analar) and the antibiotic A23187 (Calbiochem-Behring Corp.). A23187 solutions additionally contained $1 \%$ dimethyl sulfoxide (DMSO) to improve its solubility. Gain and exponent values were measured before and after different treatments and were compared by a paired 2 -tailed $t$-test. A probability of $p<0.05$ was used as the criterion for a significant change in the mean values of the parameters resulting from a treatment.

\section{Results}

Figure 2 illustrates the frequency-response function obtained by direct electrical stimulation of a tactile spine neuron in nor-
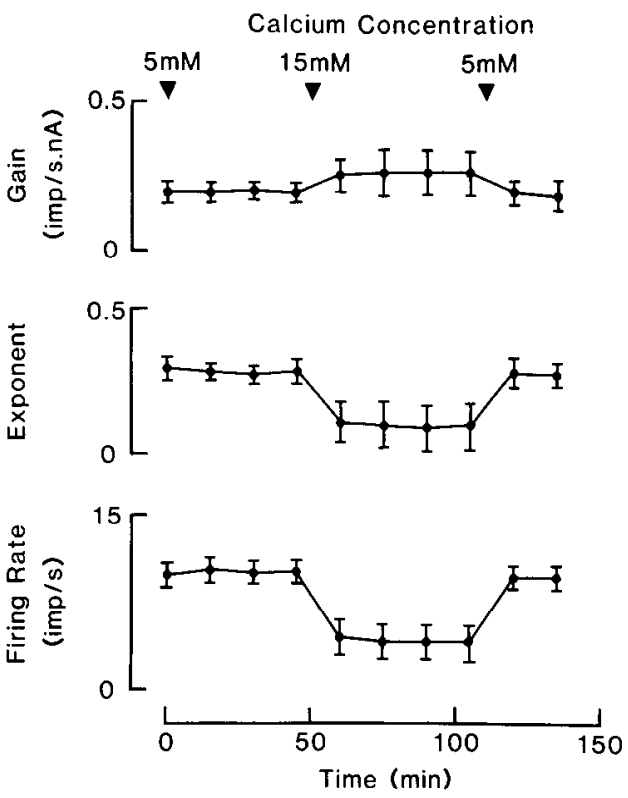

Figure 3. Dynamic behavior of tactile spine neurons as a function of time before, during, and after the application of high-calcium saline. Frequency-response functions and firing rates were measured at $15 \mathrm{~min}$ intervals. Mean values \pm SD from 25 experiments. 


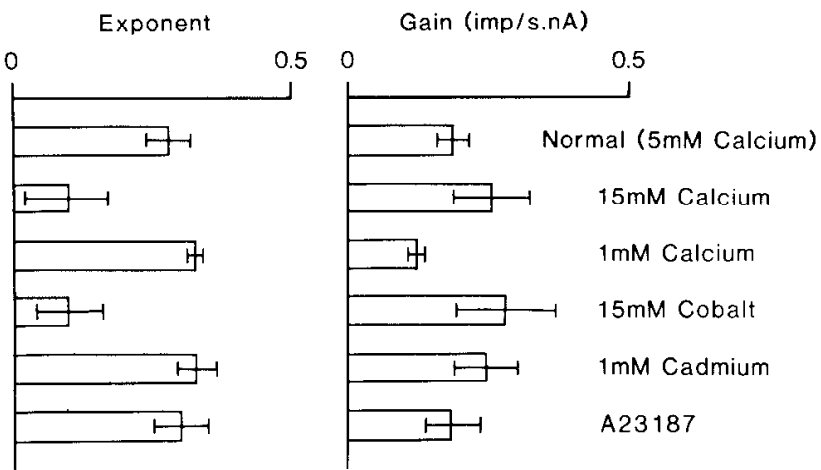

Figure 4. Bar graphs show the mean values \pm SD obtained for the exponent, $k$, and gain, $G$, from frequency-response functions under a variety of conditions. For each test condition there were 25 different animals, and 4 measurements were made from each animal, as shown in Figure 3. The bars show data pooled from all of these measurements. The data for normal saline were obtained by averaging the 4 measurements before and the 2 measurements after the high-calcium experiments, and were therefore averaged from 150 sets of values. The number of animal preparations used was 25 for each of 5 test conditions, giving a total of 125 different sensory neurons. Solutions containing cobalt, cadmium, and A23187 also contained the normal $5 \mathrm{~mm}$ calcium.

mal saline, with a calcium concentration of $5 \mathrm{~mm}$, and also after immersion for $30 \mathrm{~min}$ in a saline having a calcium concentration of $15 \mathrm{~mm}$. The response in $5 \mathrm{~mm}$ calcium showed the expected power-law form with an exponent of 0.35 and a gain of 0.15 imps/sec nA, typical values for the preparation under these conditions (French, 1984a). The increase in calcium concentration to $15 \mathrm{~mm}$ reduced the exponent to 0.015 , representing a system that was almost nonadapting. Raising the external calcium concentration therefore had a pronounced effect on the dynamic characteristics of action potential encoding by the neuron.

The tactile spine neuron is wrapped in extensive layers of glial membranes (French and Sanders, 1981), and this appears to present a significant barrier to the passage of dissolved species between the lumen of the spine and the neuron (Bernard et al., 1980). To control for errors due to this diffusion barrier, we took measurements of the frequency-response function at intervals of $15 \mathrm{~min}$ in normal saline, replaced the salinc with the test solution, and continued to measure frequency-response functions at $15 \mathrm{~min}$ intervals for $1 \mathrm{hr}$. Finally, the preparation was returned to normal saline, and 2 or more additional frequency-response functions were measured. Each frequency-response function was fitted with a power law. The gain and exponent parameters and the mean rate of action potential firing were plotted as functions of time.

The average results obtained from 25 such experiments in which the test saline contained $15 \mathrm{~mm}$ calcium showed that the change in dynamic behavior of the neuron was essentially complete after $15 \mathrm{~min}$ and remained stable for the following hour. On returning to normal saline, the response parameters also returned to normal within $15 \mathrm{~min}$ (Fig. 3). All preparations that survived for the necessary length of time showed this pattern of changes in the exponent, gain, and mean firing rates. A similar protocol was followed for salines containing low $(1 \mathrm{mM})$ calcium, cobalt (15 mM), cadmium (1 mM), DMSO (5\%), and the divalent ionophore A23187 (100 $\mu \mathrm{M}$ in 1\% DMSO). Statistical comparisons were made between the pooled measurements obtained before and after the application of the test solution. Figures 4 and 5 show bar graphs of the mean values obtained for the exponent, $k$, gain, $G$, and mean firing rate in normal saline and in 5 other test salines. Since the input noise stimulus currents were closely controlled at about $23 \mathrm{nA}$ during the experiments,

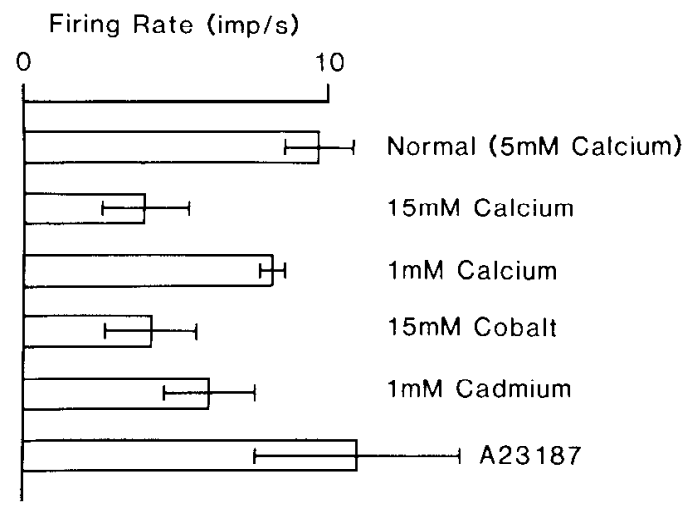

Figure 5. Bar graphs of the mean values \pm SD for the firing rates during the noise stimulation used to obtain the frequency-response functions whose data are shown in Figure 4.

the firing rates gave an indication of the excitability of the preparation under the different conditions.

High calcium increased the gain by $36 \%$ and reduced the exponent by $63 \%$, so that the sensory neuron showed less adaptation. Low calcium decreased the gain by $39 \%$ and increased the exponent by $15 \%$, representing an increase in adaptation. Both changes in calcium concentration caused a decrease in the mean firing rate, with a much larger effect in high calcium. Cobalt caused an increase in gain of $38 \%$ and a reduction in exponent of $66 \%$, similar to the changes seen with high calcium. An unpaired $t$ test of the data from the high-calcium and cobalt experiments showed no significant difference between the effects of cobalt and calcium on either the gain or the exponent. Cadmium, $1 \mathrm{~mm}$, had small but statistically significant effects on the gain and exponent.

The antibiotic A23187 is widely used to allow divalent cations to cross cell membranes and, under normal conditions, it is primarily a calcium ionophore (Carruthers and Simons, 1978). Because of the low aqueous solubility of the agent, it was necessary to dissolve it in DMSO before adding it to a saline solution; the final saline solution contained approximately $1 \%$ DMSO. In 10 control experiments a saline containing 5\% DMSO was used as the test solution. No significant differences could be seen in DMSO solutions. When the antibiotic was added to the saline, there was no dramatic change in the dynamic behavior of the neuron, apart from an increase in the variability of the firing rate. The decrease in gain was not significant, and the increase in exponent was marginally significant $(0.025<$ $p<0.05$ ).

\section{Discussion}

Power-law adaptation has now been described in a variety of arthropod mechanoreceptors and in several vertebrate systems, including mammalian touch receptors and vestibular afferents (see list in French, 1984a), suggesting that adaptation having this time course may be of general functional value and that a common mechanism may be present in many sensory afferents. Discovery of the adaptation mechanism in the tactile spine would therefore be of wide interest in sensory physiology.

If calcium ions were involved in the rapid adaptation of the tactile spine neuron, then increasing the extracellular concentration of calcium ions might be expected to increase the adaptation. However, the opposite effect was observed with the exponent, $k$, being strongly reduced and the gain, $G$, increased, reflecting less adaptation. The firing rate in high calcium was reduced to less than half its normal value under high-calcium conditions. This may seem to contradict the increasing gain parameter, but the gain measures the sensitivity to the input 
signal at a single frequency $(1 \mathrm{radian} / \mathrm{sec}$, or $0.159 \mathrm{~Hz}$ ). Inspection of Figure 2 shows that if the gain intercept were measured at a frequency of $10 \mathrm{~Hz}$ or above, it would have decreased with elevated calcium concentration instead of increasing. Since the neuron is so sensitive to high frequencies, the decreasing value of the exponent caused the total output, or firing rate, to decrease, even though the low-frequency sensitivity was increased. The firing rate measures the total output of the neuron, none of which need necessarily be correlated with the random noise stimulus.

Under the opposite condition of low calcium, the opposite effects were observed, with an increased exponent and reduced gain, corresponding to more rapid adaptation; again, this is contrary to the idea of direct calcium involvement in rapid adaptation.

Cobalt ions have been used to block calcium channels in a wide variety of excitable cell types in a range of phyla (Edwards, 1982). Cadmium ions are also effective in blocking calcium channels in some tissues, and they appear to be very specific and to act at relatively low concentrations (Adams, 1982; Cooper and Manalis, 1984; Edwards, 1982). Both cobalt and cadmium ions were found to block the after-hyperpolarization in the cockroach tactile spine neuron (French, 1985a). The family of drugs based on the dihydropyridine structure also has very specific actions on voltage-sensitive calcium channels in several tissues, but unfortunately, there is doubt about their effectiveness in neural tissues (Miller and Freedman, 1984). There are a great many binding sites for these drugs in neuronal and muscular tissues, but current evidence suggests that these binding sites are not primarily calcium channels (Schwartz et al., 1985). Under these circumstances the inorganic ions seemed the most reliable agents to use as antagonists of calcium entry in the tactile spine neuron. However, $15 \mathrm{~mm}$ cobalt, instead of opposing the action of calcium, had statistically identical effects to $15 \mathrm{~mm}$ calcium. Cadmium ions were used at a lower, but usually effective, concentration and had very little effect. The antibiotic A23187 was also ineffective in producing any changes in the dynamic properties of the neuron, although the concentration used is normally adequate to induce calcium iontophoresis (Carruthers and Simons, 1978).

The negative correlation between calcium concentration and adaptation, the similarity of the results with calcium and cobalt, and the lack of effects with cadmium and A23187 suggest that calcium and cobalt acted by a nonspccific divalent ion effect rather than by any mechanism involving a calcium channel. Frankenhaeuser and Hodgkin (1957) showed that changing the concentration of calcium ions in the extracellular solution of the squid axon caused shifts in the activation and inactivation curves for sodium and potassium ions such that high external calcium was analogous to hyperpolarization and vice versa. Although there are likely to be several mechanisms involved in this phenomenon, the major effect is probably the binding of divalent cations to the outside of the membrane, increasing the apparent potential gradient across the membrane and thus affecting the gating characteristics of the voltage-sensitive channels (Hille, 1984). In a previous study, we examined the effects of changing the membrane polarization of the tactile spine neuron by passing constant electric currents through the membrane from extracellular electrodes, and also by changing the concentration of potassium ions in the external solution (French 1984a). The results of those experiments showed that hyperpoplarizing the membrane caused a decrease in exponent, $k$, an increase in gain, $G$, and a decrease in firing rate to constant-amplitude noise stimulation. These findings were exactly analogous to the effects observed here with elevated calcium or cobalt ions in the saline. The changes in calcium concentration used here might be expected to shift ionic-channel characteristics by amounts equivalent to changes in membrane potential of about $15-20 \mathrm{mV}$
(Campbell and Hille, 1976; Frankenhacuser and Hodgkin, 1957). Although we do not have any direct data on the effects of membrane potential on the frequency response of the tactile spine neuron, comparison with our earlier results also suggests an effect equivalent to about $15 \mathrm{mV}$ change in membrane potential. The present results are therefore compatible with thc conccpt that changes in calcium concentration act primarily by a nonspecific divalent cation effect.

All of the evidence presented here is necessarily indirect, but it seems to argue against the idea that calcium entry into the cell is important for rapid adaptation in the tactile spine neuron, either through a calcium-activated potassium conductance or through any other mechanism. Although the power-law behavior of many sensory neurons has been recognized now for more than 20 years, the underlying cause remains obscure (French, 1984a; Thorson and Biederman-Thorson, 1974). The present work seems to eliminate one possible ionic mechanism underlying the power-law behavior in the tactile spine neuron.

\section{References}

Adams, P. (1982) Voltage-dependent conductances of vertebrate neurones. Trends Neurosci. 5: 116-119.

Bernard, J., J. C. Guillet, and J. P. Coillot (1980) Evidence for a barrier between blood and sensory terminal in an insect mechanoreceptor. Comp. Biochem. Physiol. A 67: 573-579.

Campbell, D. T., and B. Hille (1976) Kinetic and pharmacological properties of the sodium channel of frog skeletal muscle. J. Gen. Physiol. 67: 309-323.

Carruthers, A., and T. J. B. Simons (1978) Calcium and ionophore A23187 stimulate sugar transport in pigeon red cells. J. Physiol. (Lond.) 284: 49P.

Chapman, K. M., and R. S. Smith (1963) A linear transfer function underlying impulse frequency modulation in a cockroach mechanoreceptor. Nature 197: 699-701.

Chesler, M., and C. R. Fourtner (1981) Mechanical properties of a slow muscle in the cockroach. J. Neurobiol. 12: 391-402.

Cooper, G. P., and R. S. Manalis (1984) Cadmium: Effects on transmitter release at the frog neuromuscular junction. Eur. J. Pharmacol. 99: 251-256.

Edwards, C. (1982) The selectivity of ion channels in nerve and muscle. Neuroscience 7: 1335-1366.

Frankenhaeuser, B., and A. L. Hodgkin (1957) The action of calcium on the electrical properties of squid axons. J. Physiol. (Lond.) 137: 218-244.

French, A. S. (1973) Automated spectral analysis of neurophysiological data using intermediate magnetic tape storage. Comput. Prog. Biomed. 3: 45-57.

French, A. S. (1984a) Action potential adaptation in the femoral tactile spine of the cockroach, Periplaneta americana. J. Comp. Physiol. 155: 803-812.

French, A. S. (1984b) The receptor potential and adaptation in the cockroach tactile spine. J. Neurosci. 4: 2063-2068.

French, A. S. (1984c) The dynamic properties of the action potential encoder in an insect mechanosensory neuron. Biophys. J. 46: 285290.

French, A. S. (1985a) After-hyperpolarization and receptor potential attenuation following bursts of action potentials in an insect mechanoreceptor. Can. J. Physiol. Pharmacol. 63: 18-22.

French, A. S. (1985b) The effects of temperature on action potential encoding in the cockroach tactile spine. J. Comp. Physiol. 156: 817821 .

French, A. S., and A. V. Holden (1971) Alias-free sampling of neuronal spike trains. Kybernetik 8: 165-171.

French, A.S., and E. J. Sanders (1981) The mechanosensory apparatus of the femoral tactile spine of the cockroach, Periplaneta americana. Cell Tissue Res. 219: 53-68.

French, A. S., A. V. Holden, and R. B. Stein (1972) The estimation of the frequency response function of a mechanoreceptor. Kybernetik 11: $15-23$.

Hille, B. (1984) Ionic Channels of Excitable Membranes, Sinauer Associates, Sunderland, MA.

Jack, J. J. B., D. Noble, and R. W. Tsien (1983) Electric Current Flow in Excitable Cells, Oxford U. P., Oxford, UK. 
Jansen, J. K. S., and J. G. Nicholls (1973) Conductance changes, an electrogenic pump and the hyperpolarization of leech neurones following impulses. J. Physiol. (Lond.) 229: 635-655.

Lewis, D. V., and W. A. Wilson (1982) Calcium influx and poststimulus current during early adaptation in Aplysia giant neurons. $\mathrm{J}$ Neurophysiol. 48: 202-216.

Marmarelis, P. Z., and V. Z. Marmarelis (1978) Analysis of Physiological Systems, The White Noise Approach, Plenum, New York.

McIver, S. B. (1975) Structure of the cuticular mechanoreceptors of arthropods. Annu. Rev. Entomol. 20: 381-397.

Miller, R. J., and S. B. Freedman (1984) Are dihydropyridine binding sites voltage sensitive calcium channels? Life Sci. 34: 1205-1221.

Ottoson, D., and C. Swerup (1982) Studies on the role of calcium in adaptation of the crustacean stretch receptor. Effects of intracellular injection of calcium, EGTA and TEA. Brain Res. 244: 337-341.
Partridge, L. D., and C. F. Stevens (1976) A mechanism for spike frequency adaptation. J. Physiol. (Lond.) 256: 315-332.

Pringle, J. W. S., and V. J. Wilson (1952) The response of a sense organ to a harmonic stimulus. J. Exp. Biol. 29: 220-234.

Rice, M. J. (1975) Insect mechanoreceptor mechanisms. In Sensory Physiology and Behavior, R. Galun, P. Hillman, I. Parnas, and R. Werman, eds., pp. 135-165, Plenum, New York.

Schwartz, L. M., E. W. McCleskey, and W. Almers (1985) Dihydropyridine receptors in muscle are voltage-dependent but most are not functional calcium channels. Nature 314: 747-751.

Thomas, M. V. (1981) Properties of a $\mathrm{K}^{+}$conductance in an identified insect neurone. J. Physiol. (Lond.) 320: 117P.

Thorson, J., and M. Biederman-Thorson (1974) Distributed relaxation phenomena in sensory adaptation. Science 183: 161-172. 